Microfluidic cooling for detectors and electronics

This article has been downloaded from IOPscience. Please scroll down to see the full text article.

2012 JINST 7 C01111

(http://iopscience.iop.org/1748-0221/7/01/C01111)

View the table of contents for this issue, or go to the journal homepage for more

Download details:

IP Address: 137.138.99.125

The article was downloaded on 05/04/2012 at $15: 12$

Please note that terms and conditions apply. 


\title{
Microfluidic cooling for detectors and electronics
}

\author{
A. Mapelli, ${ }^{a, b, 1}$ P. Petagna, ${ }^{a}$ K. Howell, ${ }^{c}$ G. Nuessle ${ }^{d}$ and P. Renaud ${ }^{b}$ \\ ${ }^{a}$ CERN Physics Department, \\ Geneva, Switzerland \\ ${ }^{b}$ EPFL Microsystems Laboratory, \\ Lausanne, Switzerland \\ ${ }^{c}$ George Mason University, \\ Virginia, United States of America \\ ${ }^{d}$ Université Catholiques de Louvain \\ Louvain-la-Neuve, Belgique \\ E-mail: Alessandro.Mapelli@cern.ch
}

ABSTRACT: Micro-channel cooling is gaining considerable attention as an alternative technique for cooling of high energy physics detectors and front-end electronics. This technology is being evaluated for future tracking devices, where material budget limitations are a major concern. It is currently under investigation as an option for the cooling of the NA62 Gigatracker silicon pixel detector, where a micro-fabricated silicon cooling plate would stand directly in the beam. Other possible applications are also being studied in the context of LHC detectors upgrades. In this paper, the current status of this R\&D at CERN is presented.

KEYWORDS: Detector cooling and thermo-stabilization; Particle tracking detectors (Solid-state detectors)

\footnotetext{
${ }^{1}$ Corresponding author.
} 


\section{Contents}

1 Motivations for micro-channel cooling in HEP 1

2 Micro-fabrication technologies $\quad 3$

3 First possible HEP applications 4

3.1 NA62 GTK 5

3.2 Other possible applications 8

4 Conclusions and perspectives $\quad 9$

\section{Motivations for micro-channel cooling in HEP}

When designing the on-board cooling for High Energy Physics (HEP) tracking detector applications, the cooling efficiency should be maximized. This implies that two main issues have to be addressed:

1. The material budget should be minimized;

2. For a given quantity of heat to be removed, the temperature difference between heat source and heat sink should be minimized.

Local thermal management through silicon micro-fluidic devices (see figure 1(c)) is a good candidate for solving these issues [1]. The first point is indeed naturally addressed by the extremely limited impact of such devices in terms of material added close to the sensitive area of the detector, where only a thin structured layer of silicon is introduced, while any pipes distributing and collecting the cooling fluid can be moved in more favorable regions. The second point is dealt with by the intrinsic high cooling efficiency of micro-channel devices, for which the following heat transfer equations must be considered:

$$
\begin{aligned}
Q & =h \cdot S \cdot\left(T_{w}-T_{f}\right) \\
h & =\frac{K_{f} \cdot N u}{D_{h}},
\end{aligned}
$$

where $h$ is the heat transfer coefficient, $K_{f}$ is the fluid thermal conductivity, $N u$ is the Nusselt number (constant for a laminar confined flow), $S$ is the heat transfer surface area, $T_{w}-T_{f}$ is the temperature difference between the heat source and the heat sink and $D_{h}$ is the hydraulic diameter. The typical $D_{h}$ values, of the order of $100 \mu \mathrm{m}$, associated with micro-channel flows generate high local heat transfer coefficients. This, associated with the large heat exchange surfaces involved, allows for low temperature differences between heat source and heat sink for a given quantity of heat to be removed, $Q$. 


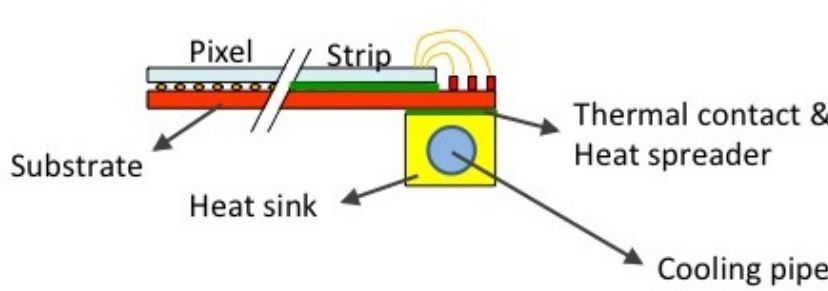

(a)

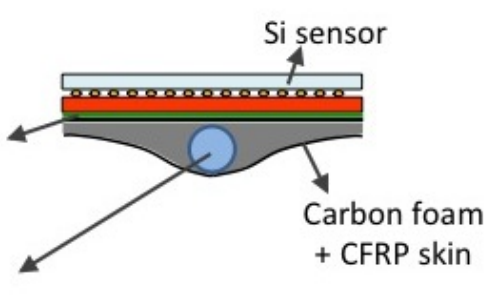

(b)

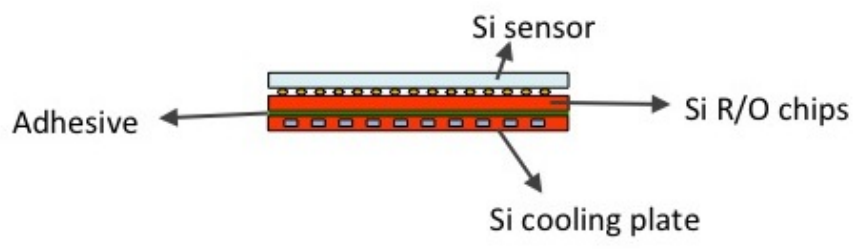

(c)

Figure 1. Cooling configurations for silicon tracking detectors.

Moreover, the cooling fluid circulates inside micro-channels structured in large area silicon wafers, placed in direct contact with the silicon surface of the detector chips or of the sensor. In this way, exploiting the high thermal conductivity of silicon and the minimization of intermediate thermal resistances, the required bulk temperature for the coolant is very close to the temperature of the heat exchange surface between the cooling plate and the heat source.

For instance, with the standard cooling approaches adopted for the large silicon trackers at the LHC, due to the small contact surfaces and the long chains of thermal resistances (see the scheme in figure 1(a)), the temperature difference $\Delta \mathrm{T}$ between the module surface and the coolant ranges typically between 15 and $20^{\circ} \mathrm{C}$ [2], while the material budget is of the order of $2 \% X_{0}$ per layer. For the sLHC phase II upgrade, a significant reduction of the material budget allocated to cooling is required. An advanced solution such as the one adopted for the ATLAS IBL [3] (see the scheme figure 1(b)) reduces the material budget by approximately half and brings the $\Delta \mathrm{T}$ down in the range $11 \div 15^{\circ} \mathrm{C}$ for a power density of $1 \mathrm{~W} / \mathrm{cm}^{2}$ [4]. As it will be detailed later, for a similar power density, a microfluidic device like the one schematized in figure $1(\mathrm{c})$ involves a $\Delta \mathrm{T}$ in the range $5 \div 7^{\circ} \mathrm{C}$ and allows for a total material budget $<0.5 \% X_{0}$ per layer, with in particular an impact of the cooling part lower than $0.15 \% X_{0}$.

The reduction of the temperature difference between the coolant and the heat source is in particular important for all future HEP applications envisaging temperatures of the silicon sensor close to $-30^{\circ} \mathrm{C}$, as the choice of the delivery temperature of the coolant has non-negligible technical impacts on the cost and complication of the required cooling plants.

Finally, the use of microfluidics silicon devices for the local thermal management of HEP tracking detectors has the additional interesting feature of using a silicon wafer as heat sink, thus eliminating the known problems of CTE mismatch between the silicon of the tracking module (chip and sensor) and the heat sink. 


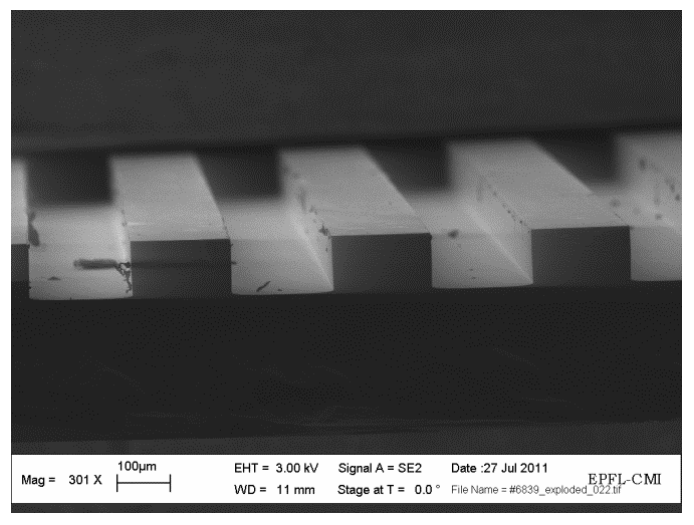

(a)

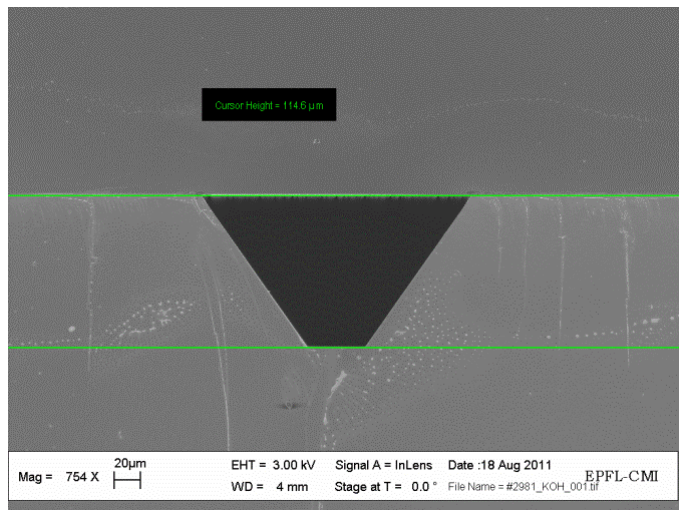

(b)

Figure 2. Silicon etched (a) by plasma to obtain vertical sidewalls and by (b) KOH to obtain sloped sidewalls.

\section{Micro-fabrication technologies}

Micro-fabrication technologies are derived from the microelectronics industry. They comprise a wide variety of techniques used for manufacturing micro-devices. In the last decades, they have been widely used to manufacture micro-electro-mechanical systems (MEMS) and microfluidic devices of interest for current investigations micro-channel cooling systems. These devices are typically made out of silicon wafers but other types of substrates are commonly used such as plastics and glasses. To fabricate a microsystem, one defines a process-flow in which many operations are performed sequentially. Common steps comprise photolithography, thin films deposition and structuration, etching, thinning by grinding and chemical mechanical polishing (CMP), bonding (at wafer level or chip level), and dicing.

Of interest for the ongoing micro-cooling projects at CERN are essentially micro-channels etched in silicon and closed with silicon wafers. The etching can be performed either in dry phase, typically with a plasma, or in wet phase. Plasma anisotropic etching results in vertical or nearvertical sidewalls independent of the crystalline orientation of the silicon wafers (see figure 2(a)). On the other hand, wet etching of silicon, in a solution of Potassium hydroxide $(\mathrm{KOH})$ for example, can lead to sloped sidewalls according to the crystalline orientation of the wafer (see figure 2(b)). The etch rates between different crystal planes differs by factors up to 200 resulting in sloped sidewalls following the crystalline planes with the slowest etch rates.

To close the cooling micro-channels reported here, two techniques are employed. Namely anodic bonding between a structured silicon wafer and an unstructured Pyrex wafer (see figure 3(a)), and direct bonding between two silicon wafers (see figure 3(b)). The first technique is well mastered at the EPFL CMi clean room. It provides a strong bond between the two wafers. However the Pyrex is the structural limiting factor as its brittleness and poor mechanical properties permit its use only at relatively low pressures, depending on the channels dimensions and geometries. On the other hand direct bonding between two silicon wafers, like Silicon Fusion Bonding for example, results in strong chemical bonds and the strength of the microfluidic is at least an order of magnitude better than for channels closed with Pyrex. But this technique cannot yet be performed 


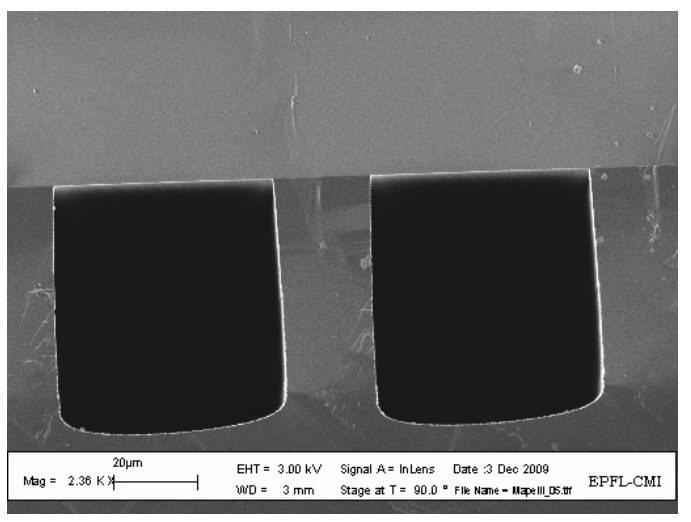

(a)

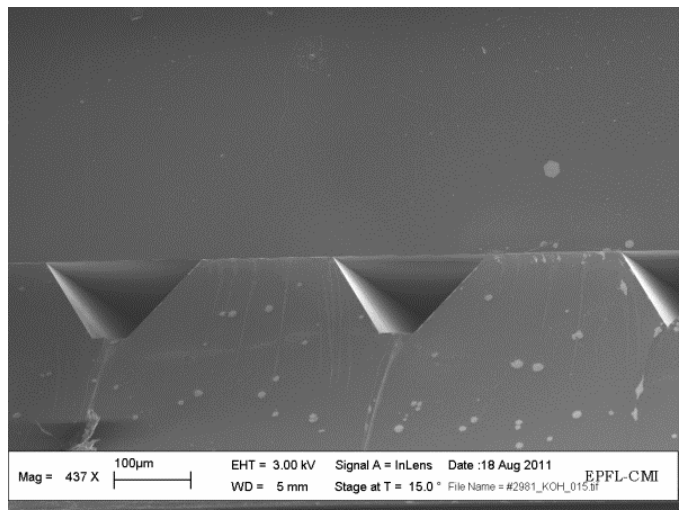

(b)

Figure 3. Silicon micro-channels closed by (a) a Pyrex wafer and (b) a silicon wafer.

reliably at EPFL. A hybrid solution has been implemented for the fabrication of the micro-channel cooling pates studied at CERN. It consists of a double anodic bonding process, where a few $\mu \mathrm{m}$ thick layer of Pyrex is interposed between two silicon wafers. In this way the structural resistance to the hydraulic pressure built into the cooling device is provided by the silicon, while the Pyrex layer only acts as a bonding agent. The very close CTE matching of Pyrex and mono-crystalline Silicon allows for safe thermal cycling within the temperature range $+40 \div-50^{\circ} \mathrm{C}$.

The microfluidic cooling devices investigated at CERN to locally remove the heat dissipated by the front-end electronics of silicon tracking detectors are fabricated with various process-flows allowing for each type of device to meet the requirements of different experiments. In all cases, the fabrication relies on the three main steps. Namely, the etching of microchannels in silicon wafers; the bonding of unstructured silicon wafers to close the micro-channels; and finally the thinning of the assembled cooling plates in the acceptance of the detectors.

\section{First possible HEP applications}

Three main lines of investigation are currently pursued. The first one, and the most advanced one, consists of an ultrathin silicon cooling plate for the Gigatracker of the NA62 [5] experiment in which hundreds of microchannels are etched. The whole acceptance area of the pixel module, which measures about $60 \times 40 \mathrm{~mm}^{2}$, must be kept uniformly cold while minimizing the material. In this case a device with uniform cooling capacity over a large surface has been designed to circulate a cold fluid in liquid phase. The second investigation is performed in the framework of the ALICE SPD upgrade [6]. In this case all the heat is produced at the periphery of a slender pixel stave, where the digital part of the electronics is located. The electronics must be kept close to room temperature, and the strategy is to remove all the material from the cooling element where it is not needed. In the end a micro-channel cooling frame provides heat extraction where the heat is produced and leaves the rest of the module with no additional material. The third configuration is the one studied as a possible alternative for the thermal management of the LHCb VeLo upgrade [7]. The cooling system of the VeLo detector is based on low temperature evaporative $\mathrm{CO}_{2}$ and the 


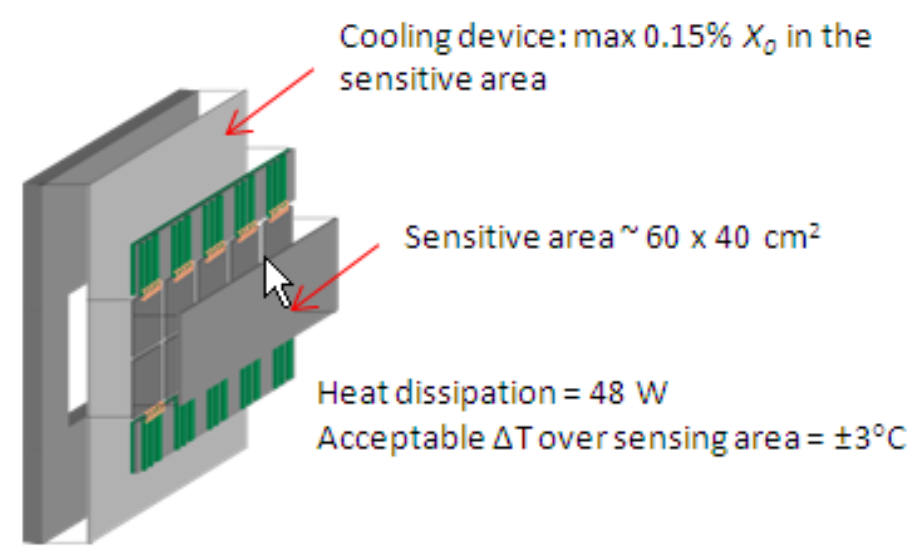

Figure 4. Schematic representation of a NA62 GTK station.

thickness limitations are not as aggressive as in the two previous detectors. Due to its peculiar thermo-fluid dynamics properties, $\mathrm{CO}_{2}$ is particularly well suited for application with microfluidics devices, allowing for channels of extremely reduced cross section, with $D_{h}$ of the order of $50 \mu \mathrm{m}$; but the pressures involved in the operation of this cooling system may be as high as 60 bars, which makes the bonding step of the fabrication a critical point for the reliability of the whole system, in particular, because the VeLo is inside the LHC secondary vacuum.

\subsection{NA62 GTK}

The first HEP application for which a microfluidic silicon device is proposed for the local thermal management of a tracking detector is the NA62 Gigatracker. In each of its three stations, this particular detector needs, among other challenging requirements, that the $48 \mathrm{~W}$ dissipated by the electronics be removed from a sensitive area of about $60 \times 40 \mathrm{~cm}^{2}$ keeping the sensor temperature always below $0^{\circ} \mathrm{C}$ and uniform in space within $\pm 3^{\circ} \mathrm{C}$ around the average, with an upper limit of $0.15 \% X_{0}$ assigned to the cooling device (see figure 4).

In figure 5, the proposed micro-fluidic silicon device is represented: $70 \mu \mathrm{m}$ deep and $200 \mu \mathrm{m}$ wide channels etched into a silicon wafer and hermetically covered with a second silicon wafer, with the assembly thinned to a total thickness of $150 \mu \mathrm{m}$ in the sensing area. A $10 \mathrm{~g} / \mathrm{s}$ flow rate of liquid $\mathrm{C}_{6} \mathrm{~F}_{14}$ is circulated in the channels, building up a pressure drop across the device of the order of 5 bars. The average material budget of the cooling device in the sensing area is $0.12 \% X_{0}$, with a maximum of $0.16 \% X_{0}$ corresponding to the walls between the channels and a minimum of $0.08 \% X_{0}$ corresponding to the channels filled up with liquid.

Prototypes of this thermal management device have been produced in the Micro-Technology Center of EPFL (figure 6) and submitted to thorough testing in realistic operational conditions at CERN (figure 7). The devices have been proved to fail at a maximum pressure of 18 bars and have been submitted to long term and cycled tests up to 12 bars in vacuum without any observable deterioration, which provides a comfortable safety margin with respect to the 6 bars maximum pressure difference to the environment expected in operation. The device presently under test has already cumulated more than 1 month of operation in varying conditions, including the simulation 


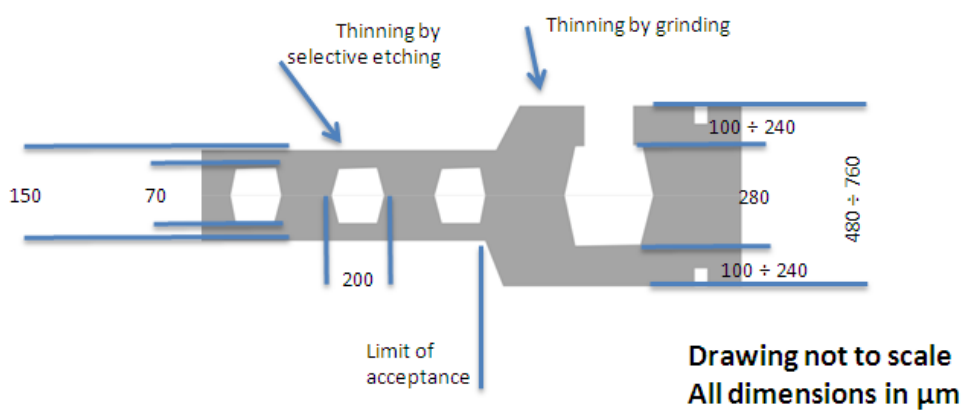

Figure 5. Proposed microfluidic devices for the GTK cooling.

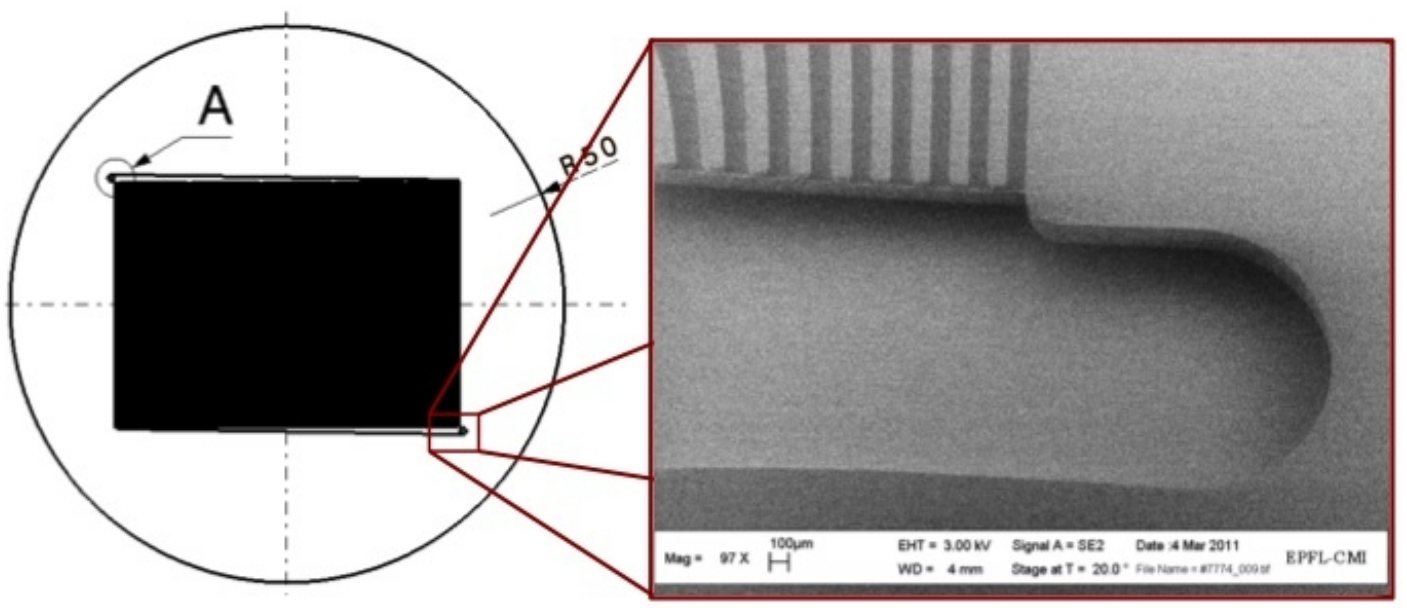

Figure 6. Drawing of the prototype microfluidic cooling device produced at EPFL CMi and Scanning Electron Microscope picture of the manifold.

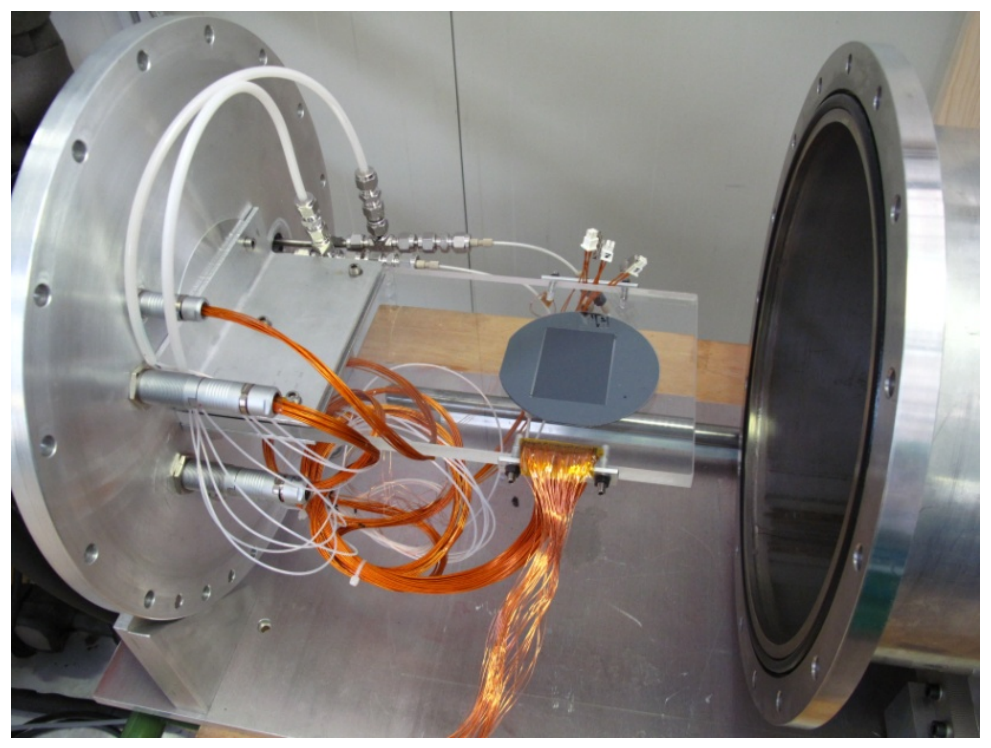

Figure 7. Prototype of the microfluidic cooling device in the vacuum vessel of the CERN experimental test-bench. 


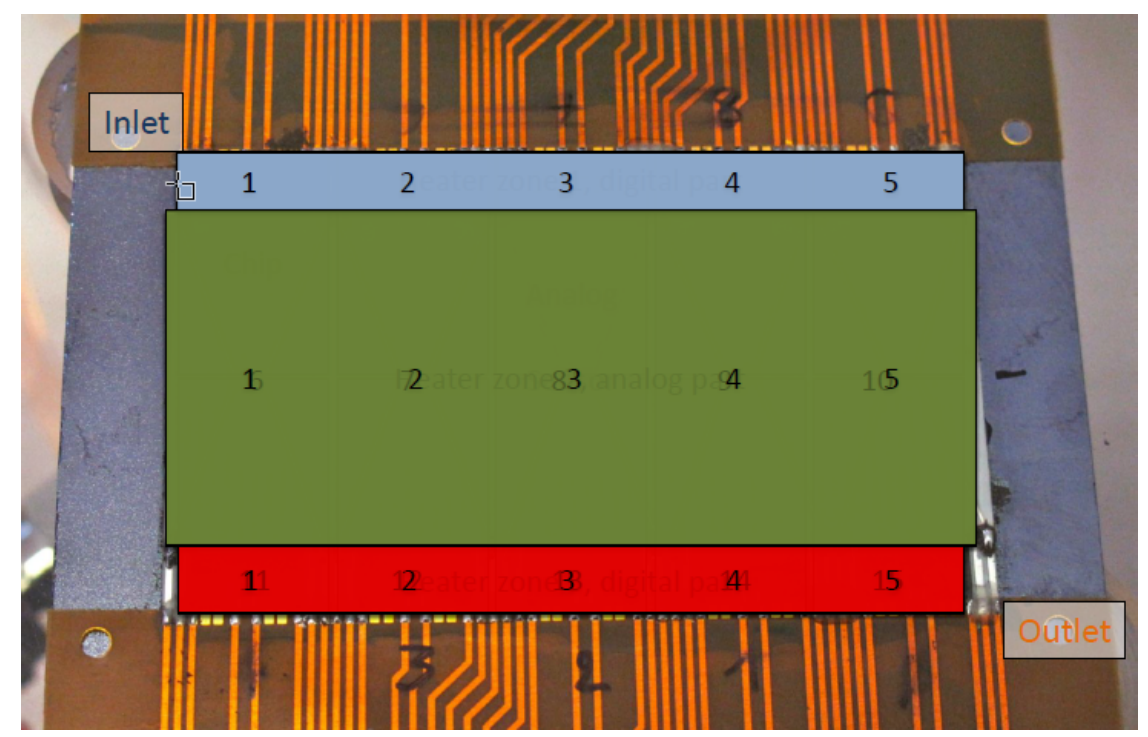

Figure 8. RTD and heater distribution on the GTK thermal mock-up. Top and bottom rows correspond to the digital parts while the central row corresponds to the analog part.

of abrupt electrics and hydraulics failures thus demonstrating its reliability for direct application for the GTK operation.

For the thermal performance tests, ceramic mock-ups of the GTK module have been produced and equipped with 20 resistive heaters - each one simulating the thermal behavior of the digital and the analog part of one chip - and 15 RTD probes to monitor the temperature distribution on the sensor surface in operational conditions. The mock-up surface has three separated heating regions, simulating the contribution of the two digital parts of the electronics, where power densities up to $4 \mathrm{~W} / \mathrm{cm}^{2}$ are expected, and the central analog part, where the power density is expected to be in the range $0.5 \div 1 \mathrm{~W} / \mathrm{cm}^{2}$ (see figure 8 ).

Figure 9 reports a typical temperature distribution on the outer surface of the mock-up $80 \%$ of the nominal power dissipation and $80 \%$ of the nominal flow rate, due to an intrinsic limitation of the cooling plant available for the test. In these tests, the fluid enters the cooling device at $-20^{\circ} \mathrm{C}$ and leaves at $-15^{\circ} \mathrm{C}$. For an estimated fluid temperature of about $-17.5^{\circ} \mathrm{C}$ in the central region of the analog part (green surface in figure 8), the temperature measured at the surface of the mock-up is comprised between $-14^{\circ} \mathrm{C}$ and $-15^{\circ} \mathrm{C}$. In this case, the temperature difference $\Delta \mathrm{T}$ between the coolant and the surface of the heater is of the order of $3^{\circ} \mathrm{C}$ in a region where the power density is about $0.8 \mathrm{~W} / \mathrm{cm}^{2}$. This shows the great potential of microfluidic cooling plates with respect to more conventional systems, including fairly advanced solutions such as the one adopted for the ATLAS IBL, mentioned in section 1 . The temperature difference $\Delta \mathrm{T}$ increases to $5 \div 8^{\circ} \mathrm{C}$ on the surface of the heaters simulating the digital part, which dissipate $4 \mathrm{~W} / \mathrm{cm}^{2}$. The temperature uniformity over the whole surface of the mock-up is better than $6^{\circ} \mathrm{C}$, which satisfies the required uniformity of $\pm 3^{\circ} \mathrm{C}$ around the average value. Moreover, the temperature uniformity over the central region of the mock-up, simulating the GTK sensor, is of the order of $1^{\circ} \mathrm{C}$. 


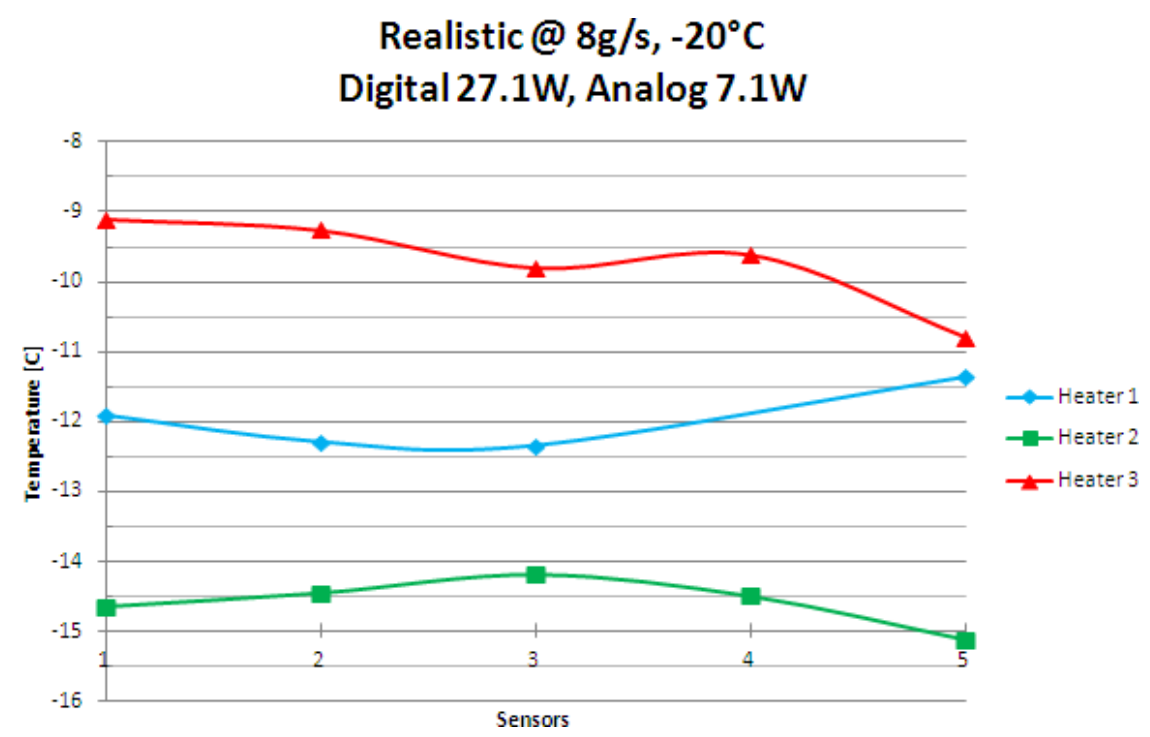

Figure 9. Example of typical thermal performance of the microfluidic cooling device.

\subsection{Other possible applications}

ALICE SPD upgrade. The heat dissipation of the silicon pixel detectors assemblies for the ALICE SPD upgrade comes exclusively from the digital part, which stands on the periphery of the pixel array (see figure 10(a)). In this case, a solution consisting of a silicon cooling frame is proposed (see figure 10(b)). It allows removing the heat produced on the outer part of the detector module with silicon microchannels without adding any addition material at the level of the acceptance area. The first prototypes of silicon micro-channel cooling frames have been produced (see figure 10(c)) and will be tested with evaporative $\mathrm{C}_{4} \mathrm{~F}_{10}$. This is the coolant currently used for the ALICE SPD. It is also envisaged to test the frames in combination with an under-pressure water cooling system.

LHCb VeLo upgrade. The present LHCb VeLo cooling system is based on evaporative $\mathrm{CO}_{2}$ and it is foreseen to keep the same cooling fluid for the upgrade. The present baseline for the thermal management of the new VeLo pixel module is based on a high conductivity substrate (possibly CVD diamond) interposed between the two detection planes and brought in contact with a cooling pipe at its periphery, out of the acceptance of the sensors [7]. However, recent studies show the great potential of silicon micro-channels for two-phase $\mathrm{CO}_{2}$ cooling systems [8] where the high pressures, up to 60 bars, required for operation are not an issue for the stability of the microfluidic system. A silicon micro-channel cooling device is under development for further studies in the context of this upgrade. It is intended to demonstrate that, by simply replacing the conductive substrate with such a system (see figure 11), one can efficiently remove heat at the level of the sensors and associated electronics for a power dissipation of $2 \mathrm{~W} / \mathrm{cm}^{2}$ with no CTE mismatch and with a maximum thickness of $250 \div 500 \mu \mathrm{m}$ in the acceptance and about $1 \mathrm{~mm}$ out of the acceptance. 


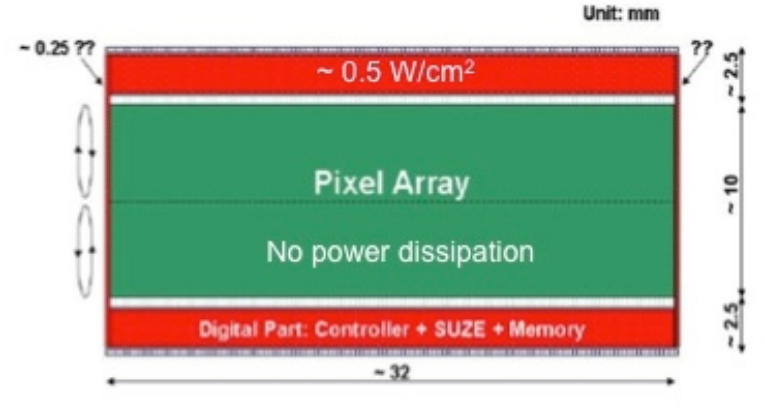

(a)

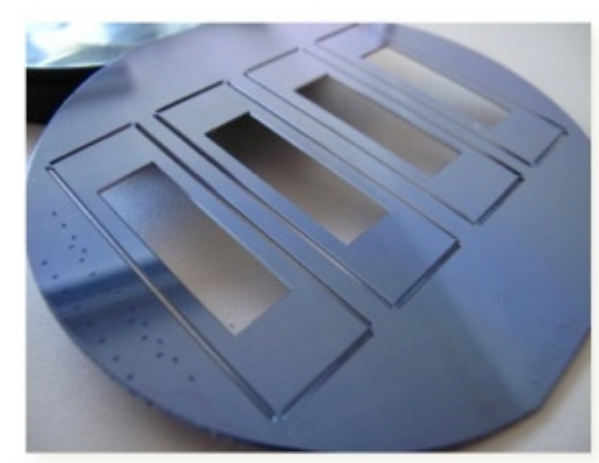

(c)

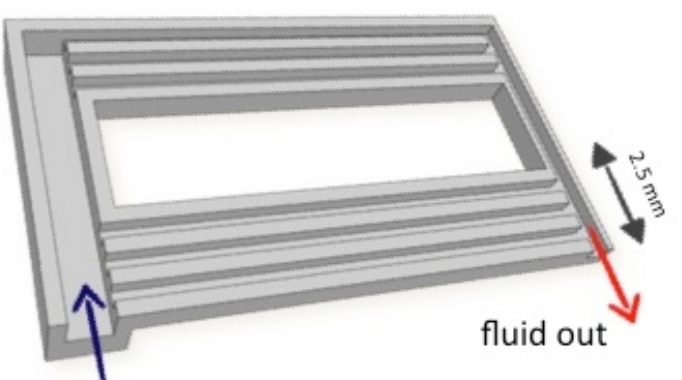

fluid in

Figure 10. (a) Drawing of the ALICE ITS upgrade pixel detector. (b) Sketch and (c) picture of the prototype cooling frames etched in 4 " silicon wafers.

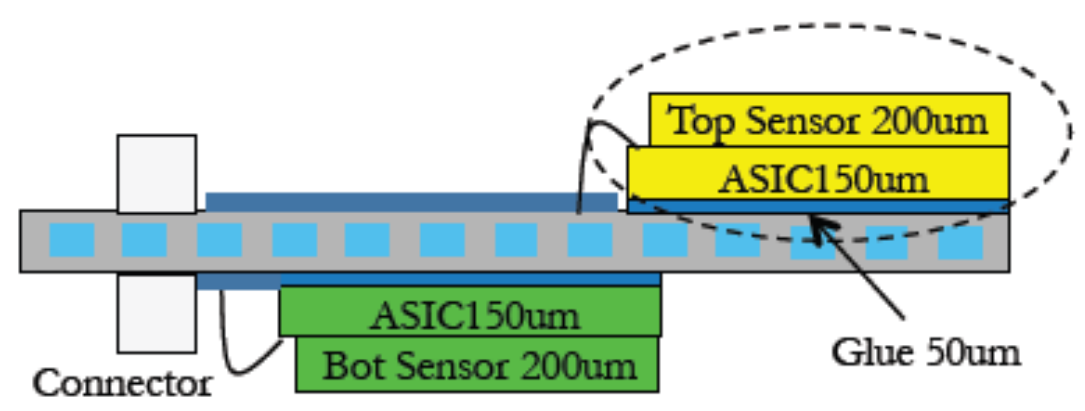

Figure 11. Drawing of a possible layout for the integration of a silicon microchannel cooling plate for the LHCb VeLo upgrade.

\section{Conclusions and perspectives}

Full silicon micro-channel cooling devices are being evaluated as a solution for the local thermal management of future tracking devices, where material budget limitations are a major concern. These cooling plates can provide evident advantages in terms of cooling efficiency, material reduction and suppression of any CTE mismatch problem.

Micro-channel devices of this type have been designed at CERN and prototyped at EPFL by well established micro-fabrication techniques: they are currently under investigation as an option for the cooling of the NA62 Gigatracker silicon pixel detector, where a micro-fabricated silicon 
cooling plate would stand directly in the beam. A thorough experimental campaign has proven that this technology provides a solution fully compliant with all the challenging technical specification set for the local thermal management of this detector.

Other possible applications are currently being studied in the context of LHC detectors upgrades, in particular for the ALICE SPD upgrade and the LHCb VeLo upgrade.

\section{Acknowledgments}

A. Pezous and P. Niedermann from CSEM. The technical staff from the EPFL CMi cleanroom.

\section{References}

[1] A. Mapelli et al., Low material budget microfabricated cooling devices for particle detectors and front-end electronics, Nucl. Phys. (Proc. Suppl.) B 215 (2011) 349.

[2] CMS collaboration et al., The CMS experiment at the CERN LHC, 2008 JINST 3 S08004.

[3] ATLAS IBL Community et al., ATLAS Insertable B-Layer Technical Design Report, CERN-LHCC-2010-013 (2010) pp. 86-110.

[4] B. Verlaat, private communication (2011).

[5] NA62 collaboration et al., NA62 Technical Design Report, NA62-10-07 (2010) pp. 123-133.

[6] ALICE ITS Upgrade collaboration et al., ALICE ITS Upgrade - Conceptual Design Report, under preparation.

[7] LHCb collaboration et al., Letter of Intent for the LHCb Upgrade, CERN-LHCC-2011-001 (2011).

[8] J. Thome et al., Two-Phase Cooling of Targets and Electronics for Particle Physics Experiments, in proceedings of TWEPP-09 Topical Workshop on Electronics for Particle Physics, Paris, France, 21-25 Sept. 2009. 